\title{
La corrupción en el Perú desde la perspectiva de los estudiantes de la Universidad Alas Peruanas - Jaén, Junio, 2013.
}

Corruption in Peru from the perspective of students Alas Peruanas University, Jaen-June, 2013.

\author{
${ }^{1}$ Ysidoro Alejandría A .
}

\section{RESUMEN}

El objetivo general es identificar la percepción que tienen los estudiantes de la Facultad de Ciencias Empresariales de la Universidad Alas Peruanas, Filial Jaén, sobre la corrupción en el Perú y su nivel de involucramiento en actos de corrupción. La metodología utilizada es inductiva-deductiva, para lo cual, se ha recurrido a un instrumento de 11 reactivos aplicada a una muestra intencional de 135 estudiantes del III y V ciclo de estudios, de una población de 385 estudiantes.

La corrupción es un flagelo generalizado que compromete, incluso a instituciones eclesiales. Desde la perspectiva de los estudiantes, la corrupción (30.84\%) es el principal problema del Perú, el Congreso de la República (22.94\%) es la institución más corrupta y la Defensoría del Pueblo (42.86\%) es la que más lucha contra este mal. El $48.89 \%$ indican no haber sido tentados por la corrupción ni se consideran corruptos, pero consideran que los actos de corrupción se dan sobre todo por coimas para agilizar trámites $(37.78 \%)$.

Los estudiantes tienen una alta conciencia de la existencia de la corrupción en el Perú y del alto nivel de involucramiento del ciudadano, por lo que la Universidad tiene el reto de consolidar un perfil profesional que garantice la acción ética con responsabilidad para una sociedad justa.

Palabras clave: Corrupción, lucha anticorrupción, involucramiento, coima.

\section{ABSTRACT}

The general objetive is to final out the perception of students of the Faculty of Business Administration of the University Alas Peruanas-Jaen, on corruption in Peru and its level of involvement in corruption. Was methodology is inductive-deductive, for which an instrument of 11 reagents was applied to a sample of 135 students of the III and V study cycle of a population of 385 students.

Corruption is a widespread scourge which compromises even to ecclesiastical institutions. From the perspective of students, corruption (30.84\%) is the main problem of Peru, the Congress of the Republic $(22.94 \%)$ is the most corrupt institution; and the ombudsman $(42.86 \%)$ combat the most this evil. The $48.89 \%$ indicate not been tempted by corruption or considered corrupt, but consider that corruption occurred mainly for bribes to speed up procedures $(37.78 \%)$.

Students have a high awareness of the existence of corruption in Peru and the high level of citizen involvement so that the university has the challenge of consolidating a professional profile that ensures ethical action with responsibility for a just society.

Keywords. Corruption, anti-corruption, involvement, bribery.

${ }^{1}$ Universidad Nacional de Jaén. Cajamarca. Perú.

a Lic. en Educación. 


\section{INTRODUCCIÓN}

Etimológicamente, la palabra corrupción proviene del vocablo latino «corrumpere»y significa "echar a perder, romper algo entre dos, destruir conjuntamente en su integridad" (Mavila 2012). Pero como fenómeno histórico ha campeado en todas las épocas y latitudes, como se puede evidenciar en el Código de Hammurabi de 1753 a.C., que habla de sanciones y condena a los que se desvían de la ley. También lo hace la Biblia, el Código de Manu e incluso la literatura griega y romana (Mavila 2012).

La corrupción se ha enraizado configurándose como parte de la cultura del pueblo, pues invade hábitos, mentes y leyes a tal punto que lo anormal se está convirtiendo en normal; y el robar es cuestión de oportunidad, dado que quien no roba peca de ingenuo, para la viveza criolla.

La situación es muy preocupante, pues son acciones delictivas que dañan seriamente la moral pública nacional, y se une al $70 \%$ de peruanos que viven bajo el temor de la delincuencia común (Huerta 2012). En este contexto, la universidad tiene que estar relacionada con el entorno, valores y avances de la modernidad, comprometida en la formación de hombres y mujeres que el Perú necesita (Maldonado 2007). Así mismo, los profesionales que se forman en las universidades, deben ser conscientes de su propia realidad, para que busquen mecanismos de lucha anticorrupción desde las aulas, y se logre construir una cultura fundada en principios éticos.

Es necesario identificar la percepción que tienen los estudiantes de la universidad sobre la corrupción en el Perú, para la toma de conciencia del entorno en que se vive y se ejerce la profesión; y sobre todo, para reflexionar sobre el nivel de implicancia de los ciudadanos en actos de corrupción.

La corrupción es el mal que más empobrecey para ayudar a despertar la conciencia social de los ciudadanos del mundo, las Naciones Unidas con Resolución 58/4 del 31/10/2003, ha proclamado el 09 de diciembre como día mundial de lucha contra la corrupción. La lucha contra la corrupción requiere mucha conciencia ciudadana porque destruye economías, debilita las instituciones, el Estado de derecho, el orden social y la confianza pública (Rodríguez 2013).También requiere "desarrollar esfuerzos concertados a nivel internacional" (PCM 2012) porque la delincuencia está organizada internacionalmente y es responsabilidad de los estados combatirla, pues impide lograr los Objetivos de Desarrollo del Milenio para mejorar la vida de millones de personas en todo el mundo (Gang 2014).

Según el ranking de Transparencia Internacional (2012) sobre la percepción de la Corrupción en el mundo, en América Latina destacan como países menos corruptos Chile y Uruguay, y como más corruptos, Haití y Venezuela. Perú, se encuentra en el puesto 80 de los países más corruptos del mundo y 15 (de 25) en América Latina, al igual que Colombia y El Salvador (IPSOS 2013). Sin embargo, el Perú ha asistido al saqueo de sus recursos desde la antigüedad. En el incanato, con el ama sua, ama quella y ama llulla, establecieron la búsqueda de la convivencia armónica (Chávarry 2014).

Los hechos de corrupción han sido registrados desde la época colonial (Villanueva 2013) y desde los años 80, en el gobierno del Presidente Alan García, la historia de corrupción se agrava "por violaciones a los derechos humanos, hiperinflación económica y actos de corrupción que superaron el nivel tolerable de este fenómeno" (Avalos 2011).

En 1977, la Declaración de Lima sobre las Líneas Básicas de la Fiscalización, son un indicador de la lucha anticorrupción. Pero, el informe de la Comisión de la Verdad, indica que 
el saqueo fue devastador durante la década de los 90 (CVR s.f.). Época en que (29 de marzo de 1996) la Organización de los Estados Americanos (con 22 países) firmara la Convención Interamericana contra la corrupción. El peruano José Miguel Insulza, fue el Secretario General de la OEA en ese entonces. El Perú se adhirió a la convención contra la corrupción en 1997 (Hampe 2014).

El primer y segundo gobierno de Fujimori Montesinos, se caracterizó por la consolidación del poder autocrático y la institucionalización de la corrupción política a nivel generalizado (Avalos 2011). Y a pesar de la recuperación de la democracia "en el país se sigue padeciendo, con gravedad crónica, de actos de corrupción en casi todas las esferas gubernamentales nacionales y regionales" (Avalos 2011).

El 31 de octubre del 2003, la lucha anticorrupción se globaliza al suscribirse la Convención de las Naciones Unidas contra la Corrupción, que fue ratificada por el Estado Peruano en el año 2004 (Oyarzún 2010). El2006, fue declarado como "Año Interamericano de la Lucha contra la Corrupción” y en el 2008, el Estado Peruano elabora el Plan Nacional de Lucha contra la Corrupción; además, mediante el DS. $N^{\circ}$ 016-2010-PCM, del 28 de enero del 2010, se crea la Comisión de Alto Nivel Anticorrupción (Oyarzún 2010).

Hoy incluso se habla de "corrupción transparente" o "delitos legales" (Sánchez, 2008). Y son considerados como más corruptos, los políticos y funcionarios públicos por el soborno, la malversación, el tráfico de influencias, el abuso del poder, el enriquecimiento ilícito, el clientelismo y la obstrucción de la justicia (IPSOS 2013). Situación que ha debilitado la credibilidad democrática, y la conciencia social de los pueblos.

El Acuerdo Nacional, dentro de sus políticas de
Estado, en su Política 26, señala "Promoción de la Ética y la Transparencia y Erradicación de la Corrupción, el Lavado de Dinero, la Evasión Tributaria y el Contrabando en todas sus formas". Asimismo, el Decreto Supremo $N^{\circ}$ 027-2007-PCM de 25 MAR 2007, en su Política 11 Anticorrupción, establece el cumplimiento obligatorio para todos y cada uno de los Ministerios y demás entidades del Gobierno Nacional (Camacho 2014).

La corrupción, es el problema principal que afecta un país pues no se respetan las leyes, se tolera que un funcionario público haga favores, se acepta dar un obsequio para agilizar un trámite; o se paga una propina para perdonar una multa y se está de acuerdo en evadir impuestos o llenar datos falsos por conveniencia. El Perú es un país donde se participa de una coima y solo el 5\% lo denuncia; y donde las instituciones más corruptas son el Congreso de la República, la Policía Nacional y el Poder Judicial (IPSOS 2013).

La corrupción tiene muchas formas para presentarse: pagos ilegales, soborno, extorsión, malversación, nepotismo, entre otros (Terrón 2013). El Banco Mundial habla incluso de la "captura del estado" para referirse al cambio de las reglas en favor de los intereses de quien está en el poder (Mavila 2012). Ser corrupto es hacer "uso indebido del poder para la obtención de un beneficio irregular, de carácter económico o no económico, a través de la violación de un deber de cumplimiento, en desmedro de la legitimidad de la autoridady de los derechos fundamentales de la persona"(PCM 2012).

"La corrupción está asociada a los pagos ilegales, el rentismo, bajo la forma de soborno, extorsión, malversación... conducta que se desvía de los deberes normales de un cargo público.....; o aquella que viola normas restrictivas de cierto tipo de influencias de beneficio privado"(Mavila 2012). 
Cuando se habla de corrupción, también se habla de moral y de ética profesional. Y se da en función pública donde no siempre se trata de dinero sino de ofertas de empleo, de ascensos y de otras ventajas (Rodríguez 2010). Así, la corrupción obstaculiza el desarrollo de los países (Moreno y Ríos 2012) pues "afecta a la economía del país y a la economía doméstica de los ciudadanos, pero también afecta derechos, acceso a servicios, afecta la gobernabilidad y la confianza en el Estado y en sus funcionarios" (PCM 2012).

La corrupción afecta a las instituciones públicas y privadas y se promueve a través del soborno (Luna 2014). Sin embargo se presenta de diferentes formas: corrupción pasiva, cuando se hace por medio de terceros $\mathrm{y} / \mathrm{o}$ aceptar promesas de ventajas para actuar de forma contraria a los deberes; la corrupción activa o hecho intencionado de prometer $\mathrm{y} / \mathrm{o}$ dar ventajas para que el funcionario actúe contra sus deberes (Mavila 2012). Es corrupción pública cuando se da en la escena política y el Estado; y es privada cuando es cometida por privados entre privados (Mavila 2012). De allí que los códigos de ética vienen cobrando importancia en la actualidad pues "condensan las normas que perfilan las conductas deseables de los miembros de las instituciones" (Mavila 2012) y las actividades empresariales encierran principios de ganar dinero dentro de la legalidad (Serrano 2014).

La corrupción es inherente al poder, por ello, afecta al sistema democrático y debilita el control (Quiroga 2012). Según Aristóteles nuestras virtudes éticas se producen y modifican por costumbre (Giraldo 2012). Entonces, existe la necesidad de maestros, hace falta la conducta ejemplar.

Ahora bien, los ciudadanos reciben servicios públicos ineficientes donde se emplea el soborno como incentivo para acceder a la prestación del mismo con mejor calidad (PCM 2012), afectando la cultura y deteriorando la calidad de vida y a pesar que el ciudadano se ve inmerso en actos de corrupción, no se considera corrupto (IPSOS 2013).

El hombre tiene una actitud ética de tolerancia de la ilegalidad y del "todo vale" (Magioncalda 2013). Y muchas veces quien se atreve a denunciar un acto de corrupción sale perjudicado. Deviniendo en desconfianza, y resquebrajamiento de los valores esenciales para la convivencia humana. Por lo que urgen los esfuerzos por la humanización del hombre desde la educación (Fisas 2013).

Es indispensable rehacer la cultura, construir un nuevo sistema de relaciones en la sociedad. Y aquí la Universidad tiene un rol fundamental para formar personas que se planteen el ejercicio de su profesión desde el compromiso con los otros y con su entorno, así como participar en la transformación de la sociedad (Salcedo 2012).

\section{MATERIALES Y MÉTODOS}

La investigación se realizó en la Facultad de Ciencias Empresariales de la Universidad Alas Peruanas Filial Jaén, específicamente con los estudiantes de Ciencias Contables y Financieras. La población estuvo conformada por 385 estudiantes, la cual se eligió intencionalmente una muestra de 135 estudiantes pertenecientes a los ciclos III, IV y V considerando los turnos de mañana, tarde y noche. Los datos fueron tomados entre el 8 al 12 de junio del 2013, a estudiantes con más de un año de permanencia en la Universidad.

En esta investigación se ha aplicado el método inductivo - deductivo, utilizando la técnica de la encuesta cuyo instrumento fue un cuestionario. Es una investigación del nivel básico descriptivo (Hernández, Fernández, \& Baptista, 2010).

El instrumento encuesta ha sido tomado de IPSOS (IPSOS 2013). Son 11 reactivos aplicados a los estudiantes de la muestra, analizados con medidas de tendencia central a través del Software SPSS 22. 


\section{RESULTADOS}

Las figuras siguientes muestran de manera estadística la percepción que tienen los estudiantes de la muestra, respecto a la corrupción en el Perú, así como su autovaloración en relación a este flagelo social.

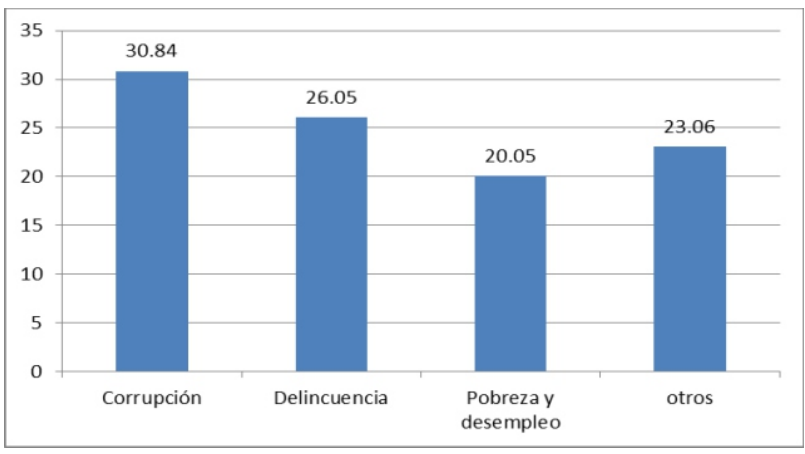

Figura1: Problema principal en el Perú

FUENTE: Encuesta aplicada a estudiantes universitarios.

En la figura 1, se puede observar que para el $30.84 \%$ de estudiantes de la muestra, la corrupción es el principal problema de nuestro país, seguido de la delincuencia en un $26.05 \%$.

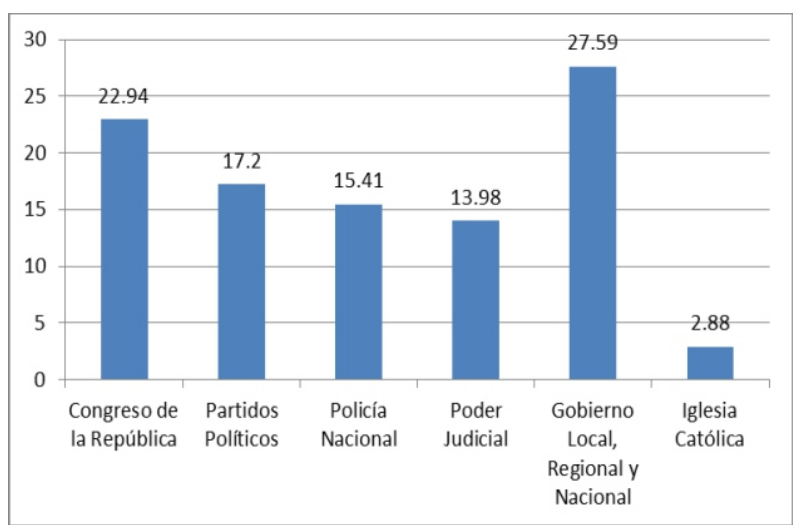

Figura2: Instituciones corruptas en el Perú

FUENTE: Encuesta aplicada a estudiantes universitarios.

La percepción de los estudiantes reflejada en la figura 2, indica que la institución más corrupta del país es el Gobierno Central, Regional y local con un $27.59 \%$, seguido del Congreso de la República con 22.94\%, los Partidos Políticos con $17.20 \%$, la Policía Nacional del Perú con $15.41 \%$, y el Poder Judicial con $13.98 \%$.

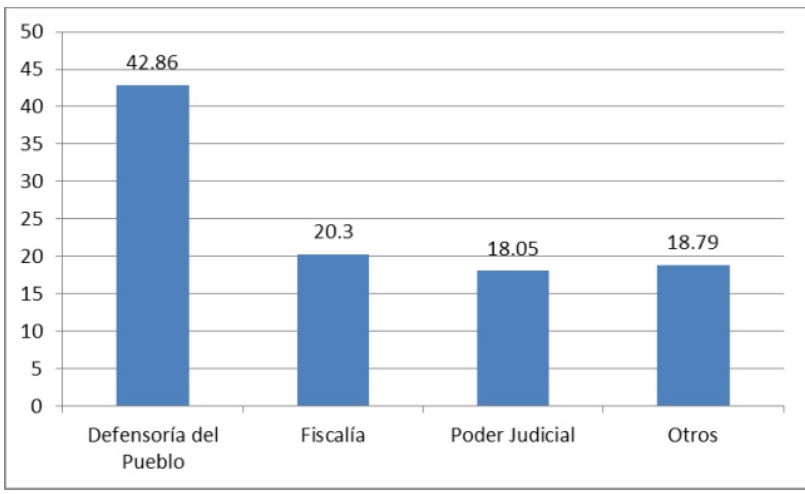

Figura 3: Instituciones que luchan contra la corrupción FUENTE: Encuesta aplicada a estudiantes universitarios.

La figura 3 indica, según los estudiantes, que la Defensoría del Pueblo es la que realiza una mayor lucha anticorrupción con $42.86 \%$, secundada por la Fiscalía con $20.30 \%$, y el Poder Judicial con $18.05 \%$.

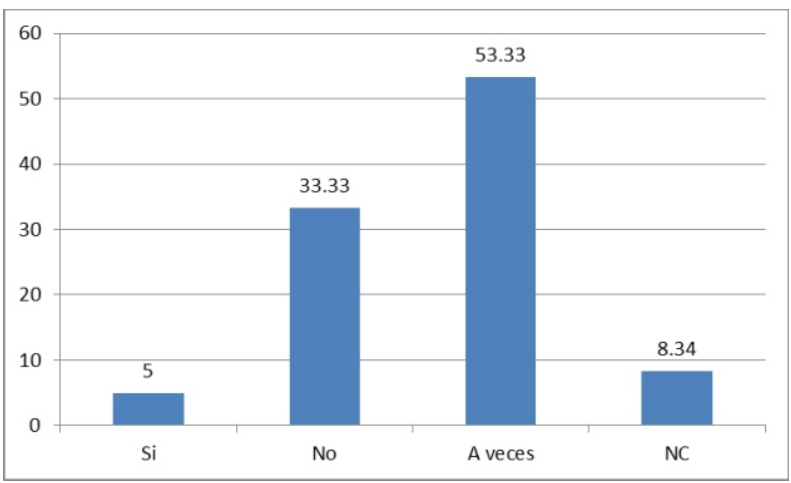

Figura 4: Cumplimiento de la ley en el Perú

FUENTE: Encuesta aplicada a estudiantes universitarios.

Respecto al cumplimiento de las leyes, el 5\% de los estudiantes indican que los peruanos si cumplen las leyes, el $33.33 \%$ que no cumplen y el $53.33 \%$ que a veces cumplen las leyes.

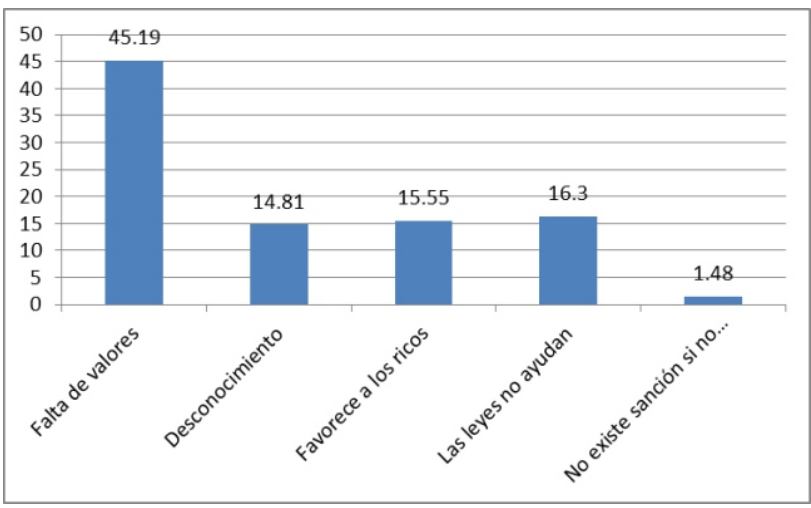

Figura 5: Motivos del incumplimiento de las leyes en el Perú FUENTE: Encuesta aplicada a estudiantes universitarios. 
La figura 5 muestra que el $45.19 \%$ de estudiantes indica que los peruanos no respetan las leyes por falta de valores, el $14.81 \%$ indica que por desconocimiento de las leyes; el $15.55 \%$ porque las leyes favorece a los ricos, no a los pobres, el $16.30 \%$ porque al respetar la ley, los procesos demoran mucho, y el 1.48\% indica que el peruano no cumple las leyes porque la sanción es leve o no existe sanción para el que incumple.

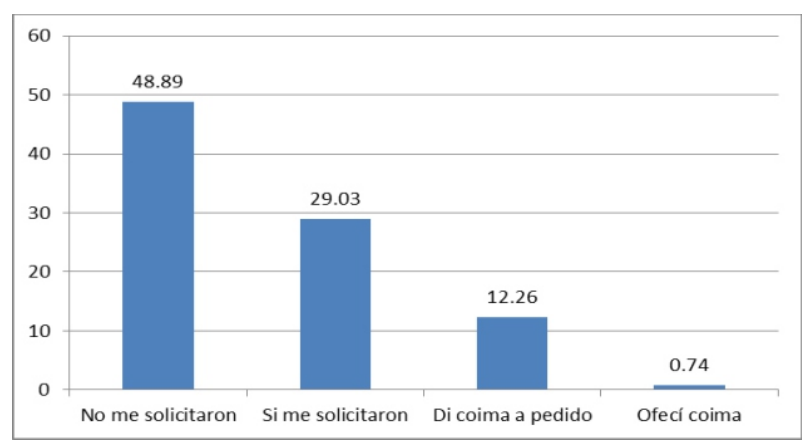

Figura 6: Involucramiento del estudiante en actos de corrupción. FUENTE: Encuesta aplicada a estudiantes universitarios.

En la figura 6, se puede observar que al $48.89 \%$ de los estudiantes nadie les solicitó coima alguna, el $29.63 \%$ si les solicitaron pero no dieron; el $12.59 \%$ indica que entregaron una coima a quien les solicitó y el $0.74 \%$ afirma que fueron ellos los que ofrecieron la coima.

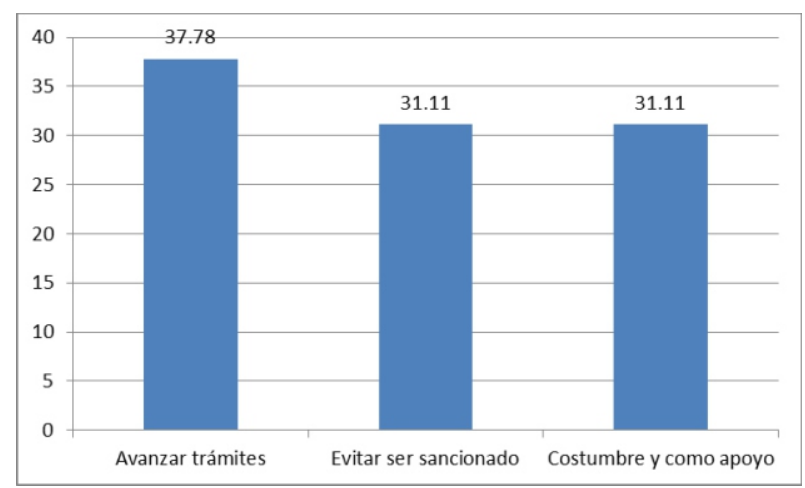

Figura 7: Motivos para entregar una coima en el Perú. FUENTE: Encuesta aplicada a estudiantes universitarios.

En la figura 7 se muestra que el $37.78 \%$ de estudiantes afirma que las coimas se dan para avanzar los trámites, el $31.11 \%$ para evitar mayores sanciones, el $31.11 \%$ porque es una costumbre y una forma de apoyar a quien te apoya.

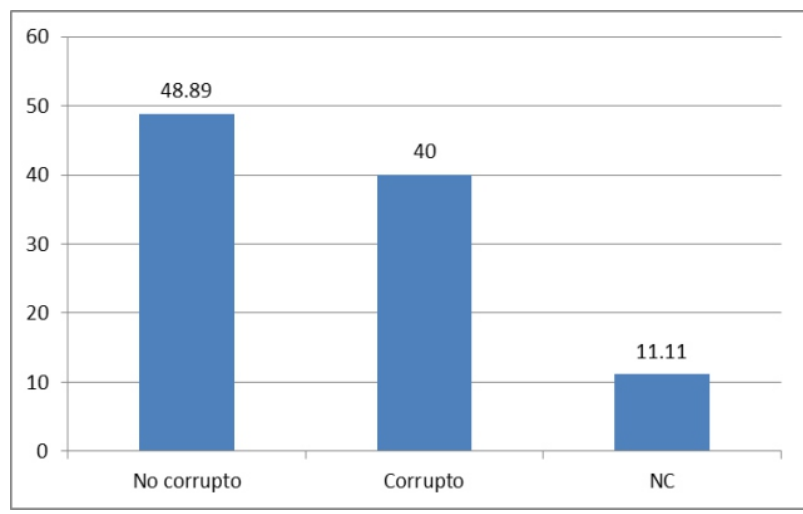

Figura 8: Motivos para entregar una coima en el Perú. FUENTE: Encuesta aplicada a estudiantes universitarios.

En la figura 8, también se puede apreciar que el $48.89 \%$ de estudiantes no se considera corrupto, el $40 \%$ sí se considera corrupto y el $11.11 \%$ no contestan.

\section{DISCUSIÓN}

Analizando la percepción sobre la corrupción en las instituciones del país que según los resultados obtenidos, se evidencia que la corrupción $(30.84 \%)$ y la delincuencia $(26.05 \%)$ son los principales problemas del país. Esto corrobora lo mostrado por IPSOS (2013) donde a la corrupción y la delincuencia como los dos principales problemas del país y donde la pobreza, las drogas, el desempleo y la contaminación son percibidos como problemas menores. Todo esto confirma que las prácticas corruptas proliferan infectando las instituciones, las iglesias y hasta los hogares (Giral 2013).

La corrupción se relaciona con el poder, pues los estudiantes consideran al Congreso de la República (22.94\%) y a los Partidos Políticos $(17.20 \%)$ como las instituciones más corruptas. Esto indica que la corrupción es pública y se da en la escena política y el estado (IPSOS 2013). O, dado que el poder corrompe, el sistema democrático es el más afectado (Quiroga 2012) pues "se afecta la confianza en las instituciones; el sistema democrático y el Derecho pierden crédito en cuanto a ser pilares educativos para la población”(Mavila 2012).

En la lucha anticorrupción, el $42.86 \%$ de los 
estudiantes, consideran a la Defensoría del Pueblo, como la embanderada de la lucha anticorrupción, concordante con los hallazgos de la Organización Transparencia a nivel nacional (IPSOS 2013). Y analizando el nivel involucramiento de la persona del estudiante en actos de corrupción, el $29.63 \%$ de estudiantes ha sido objeto de solicitud de algún tipo de coima en la sociedad. Mucho más del porcentaje que los estudiantes dan en su percepción nacional de $15 \%$, convirtiéndose esto en un acto ilícito frecuente para Jaén. Pero sin embargo, el porcentaje de involucramiento de los estudiantes en este fenómeno inmoral, es menor (12.59\%).

Las coimas se dan para que las cosas funcionen mejor $(37.78 \%)$ y para evitar sanciones (31.11\%). Los servicios no son de calidad ni oportunos contaminando la cultura popular. Solo el $48.89 \%$ de estudiantes no se considera corrupto. Existe la necesidad de una conducta ejemplar, porque la corrupción es inherente a la condición humana, es decir, el humano es corruptible (Giraldo 2012).

La actitud ante la corrupción es otro aspecto evaluado. La actitud es la postura mental del hombre frente a la acción corrupta. En el Perú, la actitud es de tolerancia de la ilegalidad y del no atreverse a luchar contra este mal. Y sobre todo porque se percibe que los delitos no son sancionados (IPSOS 2013) e incluso, si alguien denuncia es rechazado por el común de la población (Magioncalda 2013).

Con lo cual el pueblo se sumerge en falta de vivencia de los valores. Por otro lado, las actitudes humanas se inclinan a tolerar la corrupción, no denunciar e incluso justificar el soborno considerándolo como un apoyo a quien realiza el favor pues el sueldo que recibe es muy poco (IPSOS 2013).

Es indispensable, rehacer la cultura, construir un nuevo sistema de relaciones sociales. Y por ello, se requiere profesionales éticamente bien formados y socialmente comprometidos. La Universidad, ha de dar respuesta a esta urgente necesidad (Rodríguez 2014), creando y difundiendo cultura (López 2014) y participando activamente en el desarrollo del país para elevar el nivel de vida de la población.

\section{CONCLUSIONES}

Los estudiantes perciben a la corrupción como el principal problema del Perú y consideran al Congreso de la República como la institución más corrupta, y a la Defensoría del Pueblo como la institución que mejor lucha contra la corrupción.

Solo el 5\% de los estudiantes percibe que los peruanos cumplen las leyes y consideran que el incumplimiento de las leyes es por falta de valores $(45.19 \%)$.

El $48.89 \%$ de estudiantes no se ha visto involucrado en acciones de corrupción y el $37.78 \%$ precisa que la coima se da para agilizar trámites y el $31.11 \%$ indica que se da para evitar ser sancionados y es como una costumbre, como un apoyo.

A nivel de autopercepción, el $48.89 \%$ de estudiantes se considera no corrupto, pero un $40 \%$ se auto percibe que sí lo son, con lo cual se muestra las debilidades de los valores éticos y la tolerancia de la acción corrupta.

\section{AGRADECIMIENTOS.}

A los estudiantes de la Facultad de Ciencias Empresariales de la Escuela Académico Profesional de Ciencias Contables y Financieras de la Universidad Alas Peruanas Filial Jaén por su valioso aporte que permite vislumbrar tareas formativas para un futuro sostenible de los estudiantes en nuestra provincia de Jaén.

\section{REFERENCIAS BIBLIOGRÁFICAS}

Avalos, S. 2011.Corrupción en la Política, el problema de nunca acabar. http://www.diario horacero.com/pdf/informe_especial_0.pdf. 
Boehm, F. 2009. Corrupción y Anticorrupción: Una perspectiva neo institucional. (último acceso:setiembrede 2014) http://www. economiainstitucional.com/pdf/no21/ fboehm21.pdf

Chávarry, W. 2014. Moral y ética en el imperio incaico. LEX 12, n 13:303-318P.

CVR.s.f. La década de los noventa y los dos gobiernos de alberto. s.f. http://www. cverdad.org.pe/ifinal/pdf/TOMO $\% 20$ III/Cap.\% $202 \% 20$ Los $\% 20$ actores $\% 20$ polIticos $/ 2.3 \% 20 \mathrm{~L}$ A\%20DECADA\%20DEL\%2090.pdf.

Fisas, V. 2013. «Educar para una cultura de paz.» 20 de noviembre de 2013. Último acceso: 15 de noviembre de 2014. http ://www.izt. uam.mx /spring / wp-content/uploads/2013 /11/Fisas_Educar-para-una-cultura-de-paz.pdf

Gang, Q. 2014.Conferencia de Prensa Habitual Ofrecida. Acceso 15 de diciembre de 2014. http://www.fmprc.gov.cn/esp/xwfw/lxjzzdh/t12 20127.shtml.

Gestión. 2014. La otra cara de la corrupción. 06 de noviembre. Último acceso: 20 de noviembre de 2014. http://gestion.pe/opinion/editorialgestion-otra-cara-corrupcion-2113118.

Giraldo, J. 2012. Novedades en la ética de la anticorrupción. Editado por Universidad de Antioquía. Último acceso: 14 de setiembre. http://www.udea.edu.co/portal/page/portal/Bibl iotecaPortal/DetalleNoticia?p_id=69406922\&p _siteid=37.

Hampe, T. 2014. La encomienda en el Perú. 9 de Agosto. Último acceso: octubre de 2014. http://revistas.pucp.edu.pe/index.php/historica/ article/viewFile/8006/8297.

Hernández, R; Fernández, C; y Baptista, P. 2010. Metodología de la investigación. Chile: Mc Graw hill.

Huerta, F. 2012. ¿Qué determina la delincuencia en el Perú y el mundo? Editado por Instituto de Economía y Empresa S.A. 04, nº 87: 08.
Transparencia Internacional. 2012. Tabla Sintética de Resultados - IPC - 2011. Último acceso:12 de setiembre de 2013. http:// www.transparencia.org.es/indice $\% 20 \mathrm{de} \% 20$ per cepcion $\% 202011 /$ tabla_sint $\%$ c3\%89tica_de_re sultados_IPC_2011.pdf

Ipsos. 2013. VIII Encuesta Nacional sobre percepciones de la corrupción en el Perú 2013. 21 de agosto. Último acceso: 2014. http:// www.proetica.org.pe/viii-encuesta-nacionalsobre-percepciones-de-la-corrupcion-en-elperu-2013/.

López, C. 2014. http:// fido. palermo .edu /servicios_dyc/publicacionesdc/archivos/484_li bro.pdf\#page $=28$.

Luna, B. 2014. Mecanismos jurídicos contra la corrupción en la contratación estatal en colombia. Último acceso: 2014. http:// repository.urosario.edu.co/bitstream/handle/ 10336/8858/73189143-2014.pdf? sequence $=1$

Maldonado, C. 2007. La formación de la responsabilidad social en la universidad. Revista complutense de educación, ISSN 1130-2496 18, $n^{\circ} 2$.

Mavila, R. 2012. La corrupción en el poder judicial como parte del sistema de justicia en la década de 1990 - 2000: Tesis, Escuela de Post Grado, Universidad Nacional Mayor de San Marcos, Lima, 27.

Moreno, W, y Ríos,J. 2012. http://forumempresarial.uprrp.edu/volumenes/17-1/3.pdf.

Oyarzún, L.2010.Plan Anticorrupción del sector forestal y fauna silvestre.Editado por Ministerio de Agricultura.Dirección General Forestal y de Fauna Silvestre. www.pcm.gob.pe/popup_PCM /Plan anticorrupción.pdf.

PCM. DS-119-2012-PCM: Plan Nacional de lucha contra la corrupción 2012 - 2016. 09 de diciembre. http:// www. pcm.gob.pe /normaslegales/2012/DS-119-2012-PCM.pdf. 
Quiroga, M. 2012. Corrupción y gobernabilidad democrática. 25 de setiembre. http://aeg.pucp.edu.pe/corrupcion_y_gobernabi lidad_democratica.pdf.

Rodríguez,J.2013.http://online.unisc.br/seer/ind ex.php/direito/article/view/4332.

Rodríguez, L. 2010. Revista de Derecho de la Pontificia Universidad Católica de Valparaíso. http://www.rderecho.equipu.cl/index.php/rdere cho/article/view/559/527.

Rodríguez,M. 2014. Red Durango de Investigadores Educativos. http://redie.mx/ librosyrevistas/revistas/praxisinv 10.pdf \#page $=57$.

Rodríguez, Y. 2013. Plan anticorrupción. 1 de enero. http://www.puentenacionals a n t a n d e r.gov.co/a p c - a a - f i le s /65366565633665333332623331643936/plananticorrupcion-y-de-atencion-al-ciudadanoacuapuente-sa-esp.pdf.

Salcedo, J.2012. Relevancia de la ética en el ejercicio profesional. 4 de noviembre. Último acceso: 19 de noviembre de 2014. http:// www.razonypalabra.org.mx/N/N81/V81/12_Sa lcedoSalcedo_V81.pdf().

Sánchez, A. 2008. El laberinto de los Audios: una entrevista a Fernando Ampuero. Quehacer (DESCO), 45.

Serrano, F. 2014.

http://repository.unimilitar.edu.co/handle/1065 4/10799.

Terrón, A.2013. La corrupcion y sus raices: el caso filipinas.

http://openaccess.uoc.edu/webapps/o2/handle/1 0609/24642.

Villanueva, C. 2013. Los conflictos sociales y corrupción en el Perú. 03 de diciembre. http://onds.pcm.gob.pe/wp-content/uploads /2013/12/WILLAQNIKI13.pdf.

\section{CORRESPONDENCIA}

Ysidoro Alejandría Alejandría

Calle San Luis No 716- Morro Solar - Jaén, Cajamarca, Perú.

yalejandriaalejandria@unj.edu.pe.

ysidoroalejandria@gmail.com 